
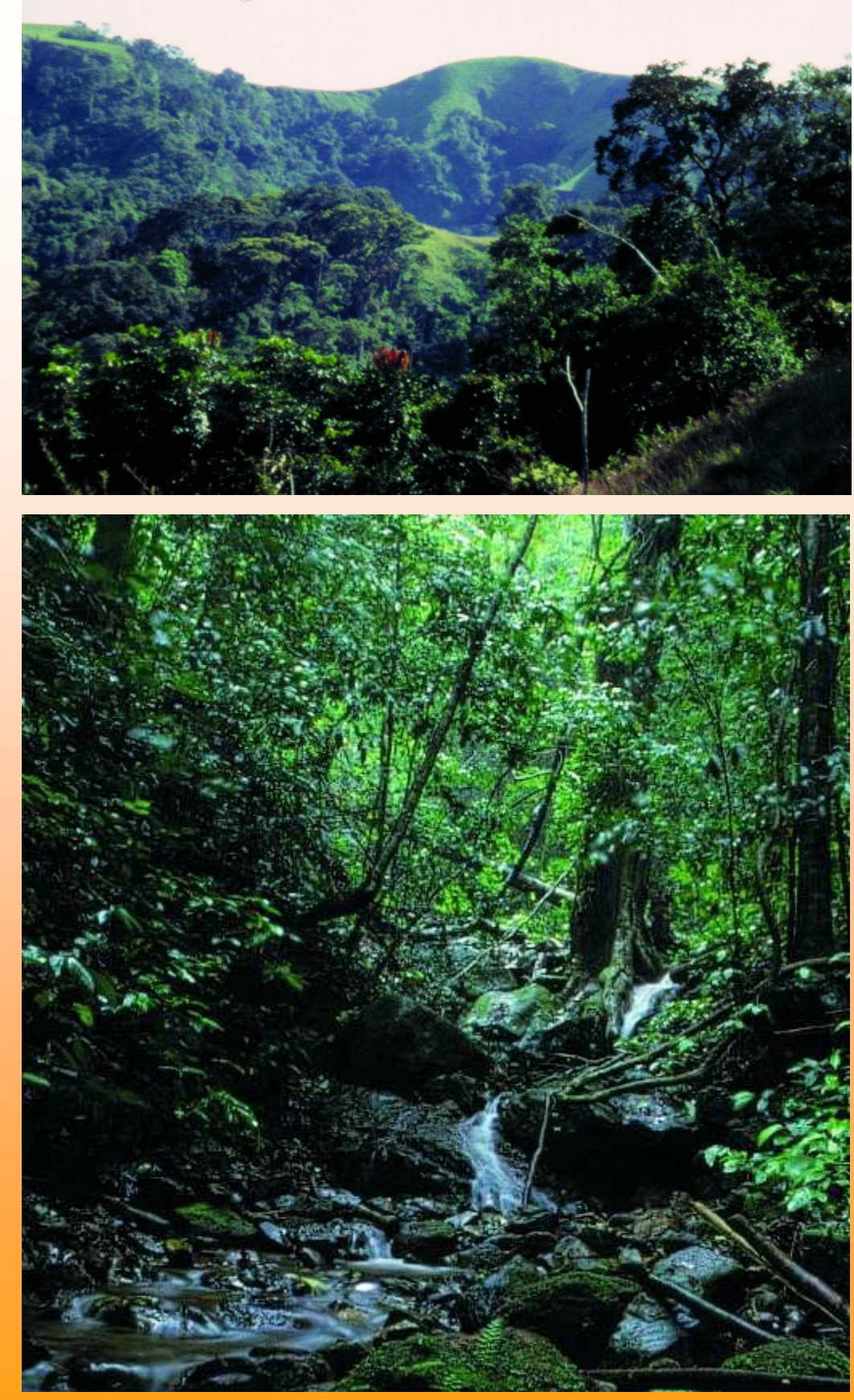

Nodulated tree legumes and their symbiotic Bradyrhizobium in African and South-American Tropical Rainforests

Y. Prin, B. Dreyfus, C. LeRoux, G. Bena, M. Diabaté, P. de Lajudie, A. Bâ, S. M. de Faria, A. Munive \& A. Galiana

Laboratoire des Symbioses Tropicales et Méditerranéennes (LSTM), Campus International de Baillarguet, 34398 Montpellier Cedex 5, France. 


\section{The Leguminosae family}

- 650 genera, 18,000 species

- Three sub-families:

- Caesalpinioideae (about $26 \%$ nodulated)

-1,900 species (woody perennials, tropics)

- Mimosoideae (about 95\% nodulated)

- 2,700 species (woody perennials, tropics)

-Papilionoideae (about $\mathbf{9 7 \%}$ nodulated)

- 13,400 species (herbaceous to trees, temperate to tropical) 

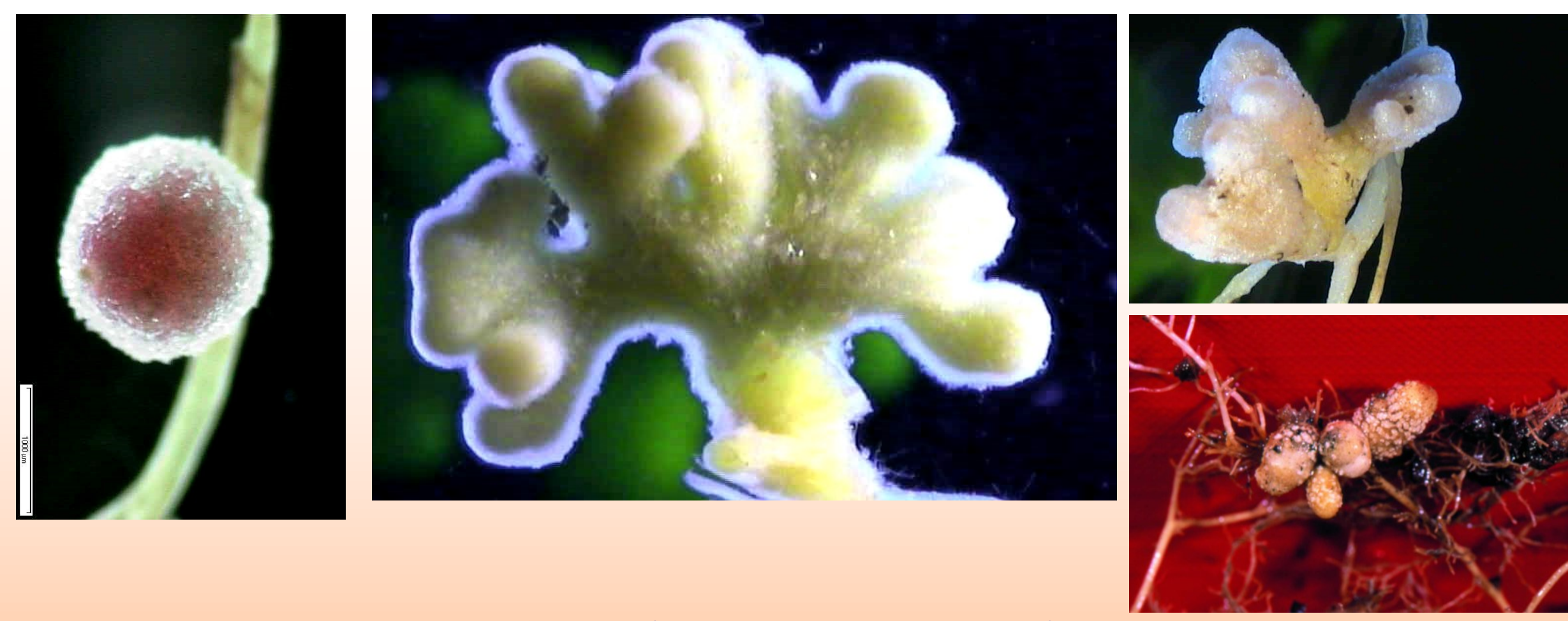

A wide diversity of nodule types in tropical rainforest trees from West Africa (Guinea) and South America (French Guyana)

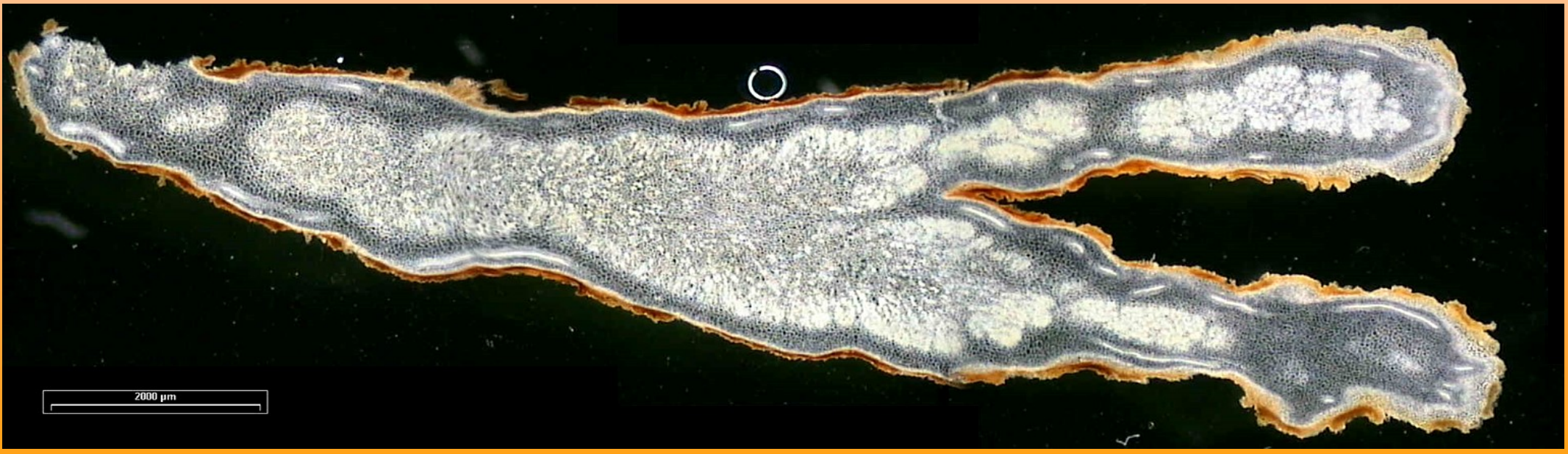




\section{Occcurence of nodulation in leguminous species of the}

primary rain forest in Guinea (West Africa)

\begin{tabular}{|c|c|c|c|c|c|}
\hline Sub-family / Tribe /Species & $\begin{array}{l}\text { Plant habit } \\
\text { and height }\end{array}$ & Location & Nodulation & $\begin{array}{c}\text { New species } \\
\text { observed }\end{array}$ & $\begin{array}{c}\text { New genus } \\
\text { observed }\end{array}$ \\
\hline \multicolumn{6}{|l|}{$\begin{array}{l}\text { CAESALPINIOIDEAE } \\
\text { CAESALPINIEAE } \\
\text { Rucenonaridantalic }\end{array}$} \\
\hline Chidlowia sanguinea & $\mathrm{T} ; 25 \mathrm{~m}$ & Ziama & + & yes & yes \\
\hline Delonix regla & $1 ; 12 \mathrm{~m}$ & NImba & $=$ & no & no \\
\hline Erythrophleum ivorense & $\mathrm{T} ; 40 \mathrm{~m}$ & Ziama & + & $\mathbf{N}$ & $\mathbf{N}$ \\
\hline Erythrophleum suaveolens & $\mathrm{T} ; 40 \mathrm{~m}$ & $\mathrm{~B}^{\circ}$ ro & + & N̦ & NÉ \\
\hline $\begin{array}{l}\text { Mezoneuron benthamianum } \\
\text { CASSIEAE }\end{array}$ & $\mathrm{S} ; 5 \mathrm{~m}$ & Dǐc k & - & yes & $\stackrel{M}{M}$ \\
\hline Cassia aubrevillei & TŹ $15 \mathrm{~m}$ & Nimba & - & yes & no \\
\hline Cassia sieberiana & Tź 15 m & $\mathrm{B}^{\circ}$ ro & - & N̦ & N̦ \\
\hline Chamaecrista mimosoides & $\mathrm{H} ; 1.5 \mathrm{~m}$ & Nimba & + & no & N̦ \\
\hline Dialium aubrevillei & $\mathrm{T} ; 30 \mathrm{~m}$ & Ziama & - & yes & $\mathbf{N}$ \\
\hline Dialium dinklagei & $\mathrm{T} ; 20 \mathrm{~m}$ & Di"c k & - & ć & $\mathbf{N}$ \\
\hline Dialium guineense & $\mathrm{T} ; 15 \mathrm{~m}$ & $\mathrm{~B}^{2}$ ro & - & no & $\stackrel{N}{N}$ \\
\hline Dialium pobeguinii & $\mathrm{T} ; 15 \mathrm{~m}$ & Ziama & - & yes & N̦ \\
\hline $\begin{array}{l}\text { Distemonanthus } \\
\text { benthamianus }\end{array}$ & $\mathrm{T} ; 35 \mathrm{~m}$ & Ziama & - & $\mathbf{N}$ & yes \\
\hline Duparquetia orchidacea & sź; 8m & Nimba & - & N̦ & N̦É \\
\hline Senna podocarpa & $\mathrm{s} ; 5 \mathrm{~m}$ & Nimba & - & N̦ & no \\
\hline $\begin{array}{l}\text { Senna siamea } \\
\text { CERCIDEAE }\end{array}$ & $\mathrm{T} ; 20 \mathrm{~m}$ & $\mathrm{~B}^{-}$ro & - & no & N̦ \\
\hline Bauhinia thonninguii & $\mathrm{T} ; 9 \mathrm{~m}$ & Pic de Fon & - & yes & no \\
\hline $\begin{array}{l}\text { Griffonia simplicifolia } \\
\text { DETARIEAE }\end{array}$ & sź; $5 \mathrm{~m}$ & 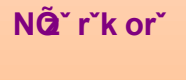 & - & N̦ & yes \\
\hline Afzelia africana & $\mathrm{T} ; 30 \mathrm{~m}$ & $\mathrm{~B}^{2}$ ro & - & no & no \\
\hline Afzelia bella var.gracilior & $\mathrm{T} ; 35 \mathrm{~m}$ & Ziama & - & yes & $\mathbf{N}$ \\
\hline Copaifera salikounda & $\mathrm{T} ; 30 \mathrm{~m}$ & Ziama & - & N & N̦ \\
\hline Daniellia ogea & $\mathrm{T} ; 30 \mathrm{~m}$ & $\mathrm{~B}^{\circ}$ ro & - & N̦É & yes \\
\hline Daniellia thurifera & $\mathrm{T} ; 35 \mathrm{~m}$ & Ziama & - & $\stackrel{N}{N}$ & $\mathbf{N}$ \\
\hline Detarium heudelotianum & $\mathrm{T} ; 20 \mathrm{~m}$ & & - & N̦ & $\mathbf{N}$ \\
\hline Detarium macrocarpum & $\mathrm{T} ; 25 \mathrm{~m}$ & Dǐc k & - & $\stackrel{N}{N}$ & NN \\
\hline Detarium senegalense & $\mathrm{T} ; 25 \mathrm{~m}$ & $\mathrm{~B}^{\circ}$ ro & - & N̦É & N̦ \\
\hline Guibourtia copallifera & $\mathrm{T} ; 25 \mathrm{~m}$ & Ziama & - & $\mathbf{N}$ & no \\
\hline Guibourtia ehie & $\mathrm{T} ; 30 \mathrm{~m}$ & Ziama & - & N̦ & N̦ \\
\hline Guibourtia leonensis & $\mathrm{T} ; 30 \mathrm{~m}$ & Dǐc k & - & N̦ & N̦ \\
\hline $\begin{array}{l}\text { Tessmannia baikiaeoides } \\
\text { AMHERSTIEAE }\end{array}$ & $\mathrm{T} ; 10 \mathrm{~m}$ & Nimba & - & $\stackrel{N}{3}$ & yes \\
\hline Anthonotha crassifolia & $\mathrm{T} ; 20 \mathrm{~m}$ & $\mathrm{~B}^{\circ}$ ro & - & yes & no \\
\hline Anthonotha fragrans & $\mathrm{T} ; 30 \mathrm{~m}$ & Ziama & - & no & $\mathbf{N}$ \\
\hline Anthonotha macrophylla & $\mathrm{T} ; 12 \mathrm{~m}$ & Dǐc k & - & yes & N̦ \\
\hline Cryptosepalum tetraphyllum & $\mathrm{T} ; 25 \mathrm{~m}$ & Ziama & - & $\mathbf{N}$ & $\dot{N}$ \\
\hline Gilbertiodendron bilineatum & $\mathrm{T} ; 15 \mathrm{~m}$ & Ziama & - & $\mathbf{N}$ & N̦ \\
\hline Gilbertiodendron limba & $\mathrm{T} ; 15 \mathrm{~m}$ & Ziama & - & Ná & N̦ \\
\hline
\end{tabular}




\section{Nodulation of leguminous species from Guinea (continued)}

\begin{tabular}{|c|c|c|c|c|c|}
\hline Sub-family / Tribe /Species & $\begin{array}{l}\text { Plant habit } \\
\text { and height }\end{array}$ & Location & Nodulation & $\begin{array}{c}\text { New } \\
\text { species } \\
\text { observed }\end{array}$ & $\begin{array}{c}\text { New } \\
\text { genus } \\
\text { observed }\end{array}$ \\
\hline \multicolumn{6}{|l|}{$\begin{array}{l}\text { MIMOSOIDEAE } \\
\text { PARKIEAE }\end{array}$} \\
\hline Parkia bicolor & $\mathrm{T} ; \mathbf{3 0} \mathrm{m}$ & Nimba & - & yes & no \\
\hline Parkia biglobosa & $\mathrm{T} ; 15 \mathrm{~m}$ & $\mathrm{~B}^{\circ}$ ro & - & no & $\mathbf{N}$ \\
\hline $\begin{array}{l}\text { Pentaclethra macrophylla } \\
\text { MIMOSEAE }\end{array}$ & $\mathrm{T} ; 25 \mathrm{~m}$ & Ziama & + & N̦ & $\underset{N}{N}$ \\
\hline Adenopodia scelerata & $\mathrm{V} ; \mathbf{3 0} \mathrm{m}$ & Nimba & - & yes & yes \\
\hline Aubrevillea kerstingii & $\mathrm{T} ; 30 \mathrm{~m}$ & Ziama & - & $\mathbf{N}$ & $\mathbf{N}$ \\
\hline Aubrevillea platycarpa & $\mathrm{T} ; 30 \mathrm{~m}$ & Ziama & + & yes & yes \\
\hline calpucalyx aublevillet & 1420111 & LIamia & & Ţ, & 110 \\
\hline Calpocalyx brevibracteatus & $\mathrm{T} ; 25 \mathrm{~m}$ & Ziama & - & $\mathbf{N}$ & $\mathbf{N}$ \\
\hline Dichrostachys cinerea & $\mathrm{S} ; 8 \mathrm{~m}$ & $\mathrm{~B}^{2}$ ro & + & no & N̦É \\
\hline Entada africana & TŹ $12 \mathrm{~m}$ & $B^{2}$ ro & + & yes & N̦ \\
\hline Entada gigas & VŹ; 30 m & Dǐck k & + & N̦É & $\mathbf{N}$ \\
\hline Entada mannii & $\mathrm{s} ; 15 \mathrm{~m}$ & Ziama & + & N̦ & N̦ \\
\hline Mimosa invisa & $\mathrm{s} ; 0.5 \mathrm{~m}$ & $\mathrm{Di}^{\circ} \mathrm{ck}^{\circ}$ & + & no & N̦ \\
\hline Newtonia aubrevillei & $\mathrm{T} ; 30 \mathrm{~m}$ & Ziama & - & yes & N̦ \\
\hline Newtonia duparquetiana & TŹ $25 \mathrm{~m}$ & Nimba & - & $\mathbf{N}$ & $\mathbf{N}$ \\
\hline Piptadeniastrum africanum & TŹ $40 \mathrm{~m}$ & Ziama & + & no & no \\
\hline Tetrapleura tetraptera & $\mathrm{T} ; 20 \mathrm{~m}$ & Ziama & + & yes & yes \\
\hline xylla evansII & $1 ; 25 \mathrm{~m}$ & DICK K & + & N̦ & no \\
\hline \multicolumn{6}{|l|}{ INGEAE } \\
\hline Albizia adianthifolia & $\mathrm{T} ; 20 \mathrm{~m}$ & Dǐc k & + & no & no \\
\hline Albizia altissima & $\mathrm{T} ; 20 \mathrm{~m}$ & Ziama & + & yes & N̦ \\
\hline Albizia ferruginea & $\mathrm{T} ; 20 \mathrm{~m}$ & $\mathrm{~B}^{2}$ ro & + & no & N̦ \\
\hline Albizia glaberrima & $\mathrm{T} ; 15 \mathrm{~m}$ & Nimba & + & $\mathbf{N}$ & $\mathbf{N}$ \\
\hline Albizia zygia & $\mathrm{T} ; 20 \mathrm{~m}$ & Ziama & + & $\stackrel{N}{N}$ & $\mathbf{N}$ \\
\hline Samanea dinklagei & $\mathrm{T} ; 20 \mathrm{~m}$ & Ziama & + & yes & $\mathbf{N}$ \\
\hline
\end{tabular}


Nodulation of leguminous species from Guinea (continued)

\begin{tabular}{|c|c|c|c|c|c|}
\hline Sub-family / Tribe /Species & $\begin{array}{l}\text { Plant habit } \\
\text { and height }\end{array}$ & Location & Nodulation & $\begin{array}{l}\text { New species } \\
\text { observed }\end{array}$ & $\begin{array}{c}\text { New genus } \\
\text { observed }\end{array}$ \\
\hline $\begin{array}{l}\text { PAPILIONOIDEAE } \\
\text { SWARTZIEAE } \\
\text { Bobgunnia fistuloides } \\
\text { SOPHOREAF }\end{array}$ & $\mathrm{T} ; 15 \mathrm{~m}$ & Dǐc ǩ & - & yes & no \\
\hline \multicolumn{2}{|c|}{ Amphimas pterocarpoides } & & + & yes & yes \\
\hline Baphia nitida & $\mathrm{T} ; 10 \mathrm{~m}$ & Nimba & + & no & no \\
\hline $\begin{array}{l}\text { Baphia capparidifolia } \\
\text { DALBERGIEAE }\end{array}$ & $\mathrm{T} ; 8 \mathrm{~m}$ & $\mathrm{Di}^{\circ} \mathrm{ck}^{\circ}$ & + & yes & N̦ \\
\hline Dalbergia afzeliana & $\mathrm{V} ; 35 \mathrm{~m}$ & Nimba & - & yes & no \\
\hline Dalbergia bignonae & $V ; 25 \mathrm{~m}$ & $\mathrm{~B}^{\circ}$ ro & + & $\mathbf{N}$ & $\mathbf{N}$ \\
\hline Dalbergia hostilis & $v ; 15 \mathrm{~m}$ & Ziama & + & $\stackrel{\text { N̦ }}{2}$ & $\stackrel{3}{N}$ \\
\hline Dalhoretia ohlanoifalia & c. $\mathrm{V} \cdot 10 \mathrm{~m}$ & nïnk" & & $\mathbf{N}$ & $\mathbf{N}$ \\
\hline \multicolumn{2}{|c|}{ Pterocarpus erinaceus } & & + & & \\
\hline \multicolumn{2}{|c|}{ Pterocarpus mildbraedii } & & + & & \\
\hline \multicolumn{2}{|c|}{ Pterocarpus santalinoides } & & - & & \\
\hline MILLE IIIIEAE & & & & & \\
\hline Dalbergiella welwitschii & $S ; V ; 20 \mathrm{~m}$ & Nimba & - & yes & no \\
\hline Leptoderris brachyptera & $\mathrm{S} ; \mathrm{V} ; 20 \mathrm{~m}$ & Ziama & + & $\mathbf{N}$ & $\mathbf{N}$ \\
\hline Leptoderris fasciculata & $\mathrm{S} ; \mathrm{V} ; 20 \mathrm{~m}$ & Nimba & + & $\mathbf{N}$ & $\mathbf{N}$ \\
\hline Lonchocarpus cyanescens & $\mathrm{S} ; \mathrm{V} ; 30 \mathrm{~m}$ & Nimba & - & $\ddot{N}$ & $\ddot{N}$ \\
\hline Millettia dinklagei & $S ; V ; 15 \mathrm{~m}$ & Nimba & + & N̦ & N̦ \\
\hline Millettia griffoniana & $\mathrm{T} ; 15 \mathrm{~m}$ & Nimba & - & $\mathbf{N}$ & $\mathbf{N}$ \\
\hline Millettia lucens & $S ; V ; 15 \mathrm{~m}$ & $B^{-}$ro & - & N̦ & 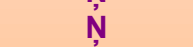 \\
\hline Millettia rhodantha & $\mathrm{T} ; 12 \mathrm{~m}$ & Ziama & + & $\mathbf{N}$ & N \\
\hline Millettia zechiana & $\mathrm{s} ; 10 \mathrm{~m}$ & Ziama & + & $\stackrel{N}{N}$ & $\vec{N}$ \\
\hline Platysepalum hirsutum & $\mathrm{S} ; \mathrm{V} ; 20 \mathrm{~m}$ & Nimba & - & N̦ & yes \\
\hline $\begin{array}{l}\text { Tephrosia flexuosa } \\
\text { DESMODIEAE }\end{array}$ & $\mathrm{s} ; 1.5 \mathrm{~m}$ & Nimba & - & $\stackrel{\text { N }}{3}$ & no \\
\hline $\begin{array}{l}\text { Droogmansia scaettaiana } \\
\text { PHASEOLEAE }\end{array}$ & $\mathrm{H} ; 2 \mathrm{~m}$ & Nimba & + & yes & no \\
\hline Dioclea reflexa & $S ; V ; 15 \mathrm{~m}$ & Nimba & - & no & no \\
\hline Dolichos dinklagei & $\mathrm{s} ; 1.5 \mathrm{~m}$ & Nimba & - & yes & $\mathbf{N}$ \\
\hline Dolichos tonkouiensis & $s z ; ; 15 \mathrm{~m}$ & Nimba & + & N̦ & $\stackrel{N}{N}$ \\
\hline Erythrina milbraedii & $\mathrm{T} ; 30 \mathrm{~m}$ & Ziama & + & N̦ & N̦ \\
\hline Erythrina senegalensis & $\mathrm{S} ; 8 \mathrm{~m}$ & Dǐc k & + & no & $\mathbf{N}$ \\
\hline Mucuna flagellipes & sź;VŹ;5 m & $B$ ro & - & yes & $\stackrel{N}{N}$ \\
\hline Mucuna poggei & $\mathrm{S} ; \mathrm{V} ; 20 \mathrm{~m}$ & $\mathrm{~B}^{2}$ ro & + & no & N̦ \\
\hline $\begin{array}{c}\text { Physostigma venenosum } \\
\text { AESCHYN OMENEAE }\end{array}$ & $\mathrm{s} ; \mathrm{v} ; 6 \mathrm{~m}$ & $\mathrm{~B}^{2}$ ro & - & yes & yes \\
\hline Kotschya lutea & $\mathrm{S} ; 2 \mathrm{~m}$ & Nimba & + & yes & no \\
\hline Kotschya ochreata & $\mathrm{S} ; 2.5 \mathrm{~m}$ & Nimba & + & $\mathbf{N}$ & $\mathbf{N}$ \\
\hline $\begin{array}{l}\text { Ormocarpum megalophyllum } \\
\text { CROTALARIEAE }\end{array}$ & $\mathrm{s} ; 1.5 \mathrm{~m}$ & Nimba & - & $\stackrel{\text { Ņ }}{ }$ & $\stackrel{\text { Ņ }}{ }$ \\
\hline Crotalaria lathyroides & $\mathrm{s} ; 2 \mathrm{~m}$ & $\mathrm{~B}^{\circ}$ ro & + & no & $\mathbf{N}$ \\
\hline
\end{tabular}


Occurence of nodulation in leguminous tree species of the Amazonian bassin (French Guyana)

\section{Sub-family / Tribe /Species \\ Nodulation New species}

observed

CAESALPINIOIDEAE CAESALPINIEAE

Dimorphandra polyandra

Tachigali melinonii

Tachigali myrmecophila

$$
\text { CASSIEAE }
$$

Chamaecrista diphylla

$$
\text { DETARIAE }
$$

Macrolobium bifolium

MIMOSOIDEAE

\section{MIMOSEAE}

Stryphnodendron

pulcherrimum

$$
\text { INGEAE }
$$

Hydrochorea corymbosa

Inga disticha

Inga sertulifera

Zygia latifolia

Zygia cataracteae

PAPILIONOIDEAE

\section{SWARTZIEAE}

Bocoa prouacensis

Swartzia polyphylla

SOPHOREAE

Diplotropis purpurea

Ormosia coutinhoi

Spirotropis longifolia

Andira inermis

Dalbergia monetaria

Hymenolobium flavum

Machaerium Iunatum

Paramachaerium

ormososioides

Pterocarpus officinalis

Vatairea erythrocarpa

BRONGNIARTIEAE

Poecilanthe hostmannii

PHASEOLEAE

Clitoria fairchildiana

Erythrina amazonica

$\begin{array}{ccc}+ & \text { yes } & \text { no } \\ + & \text { no } & N \\ + & \text { yes } & \text { N̦ } \\ & & \\ + & \text { no } & \text { no } \\ & & \\ & \text { yes } & \text { no } \\ & & \\ + & \text { no } & \text { no } \\ & & \\ + & \text { no } & \text { no } \\ + & \text { N } & N \\ + & \text { yes } & N \\ + & \text { no } & N \\ + & N & N\end{array}$

no

$\stackrel{N}{N}$

no

no

no 


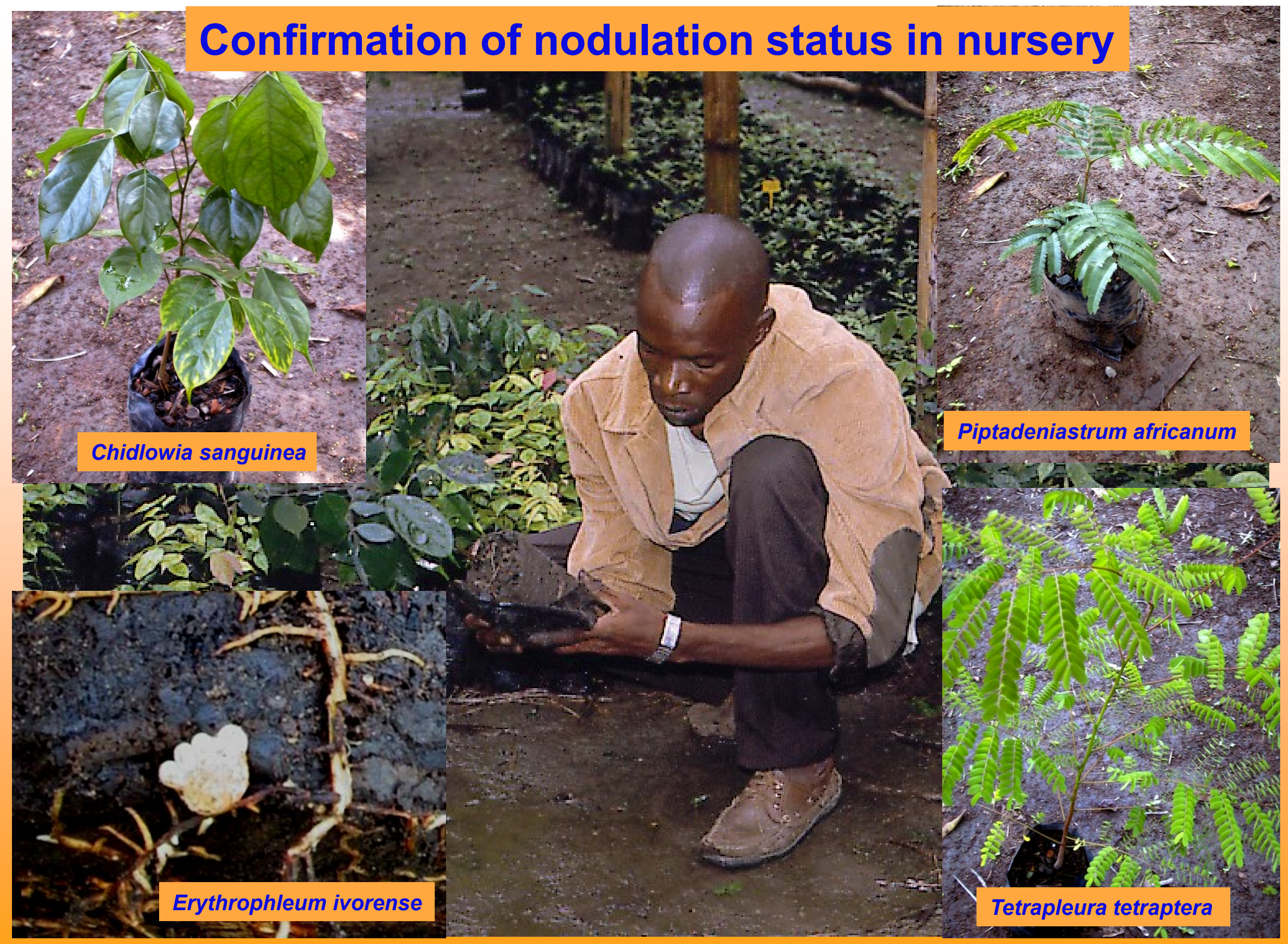




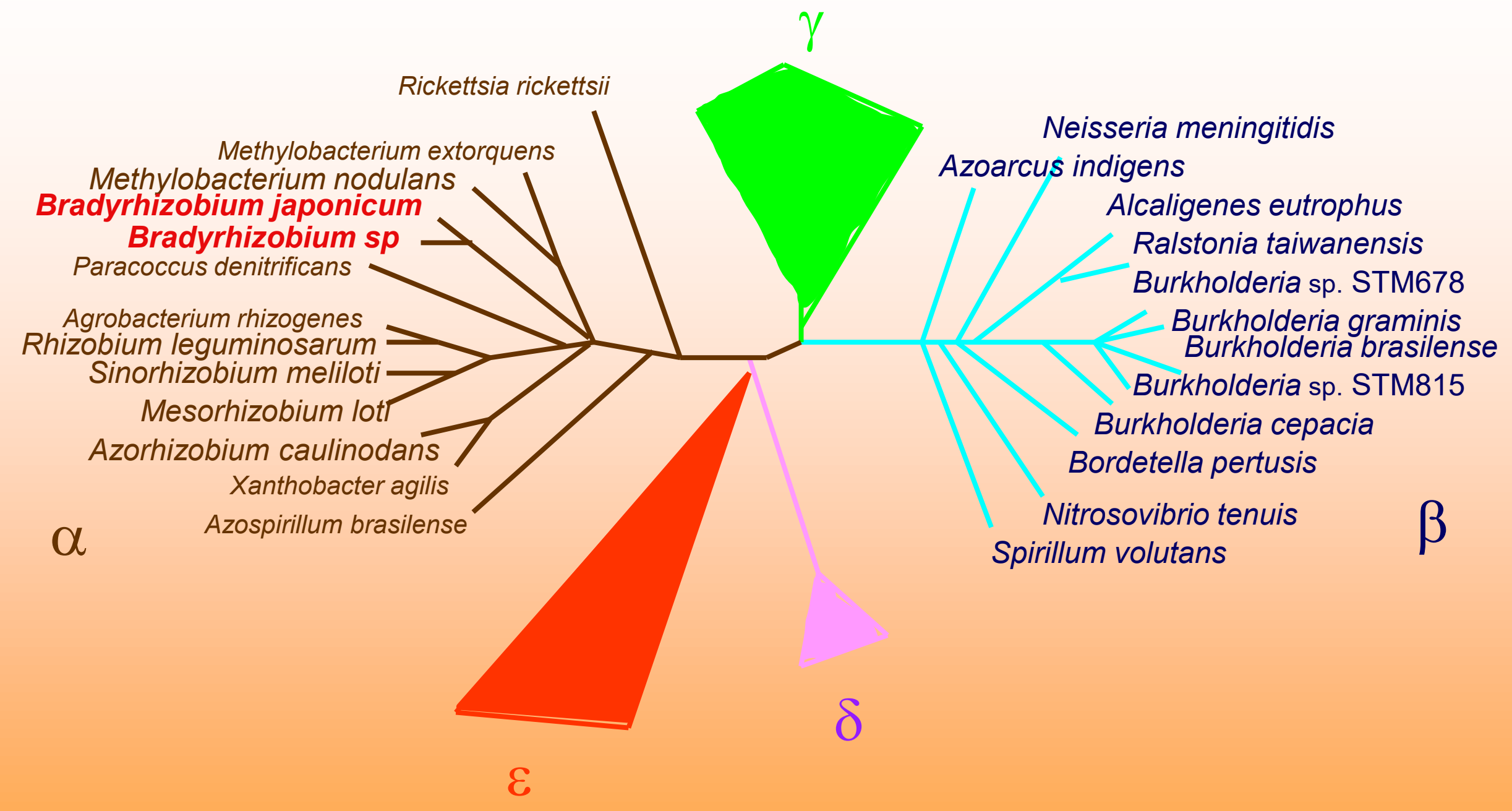

\section{Phylogeny of $16 S$ rDNA}

- 0.01 changes 


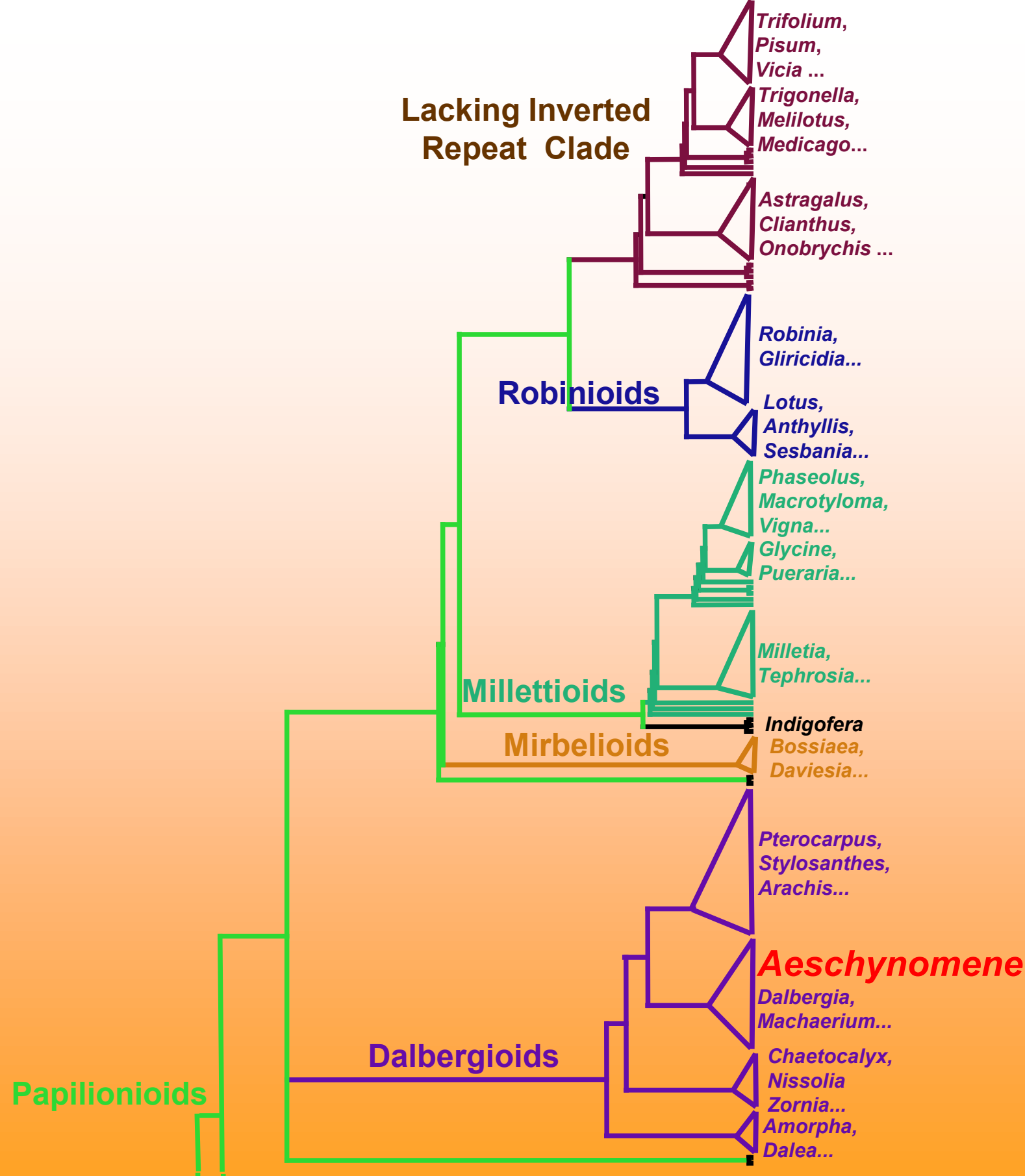

Phylogeny of Leguminosae based on parsimony analyses of matK gene sequences, adapted from Wojciechowski et al. (2004) 
Plant Genus species: Amazonian species Plant Genus species: Guinean species

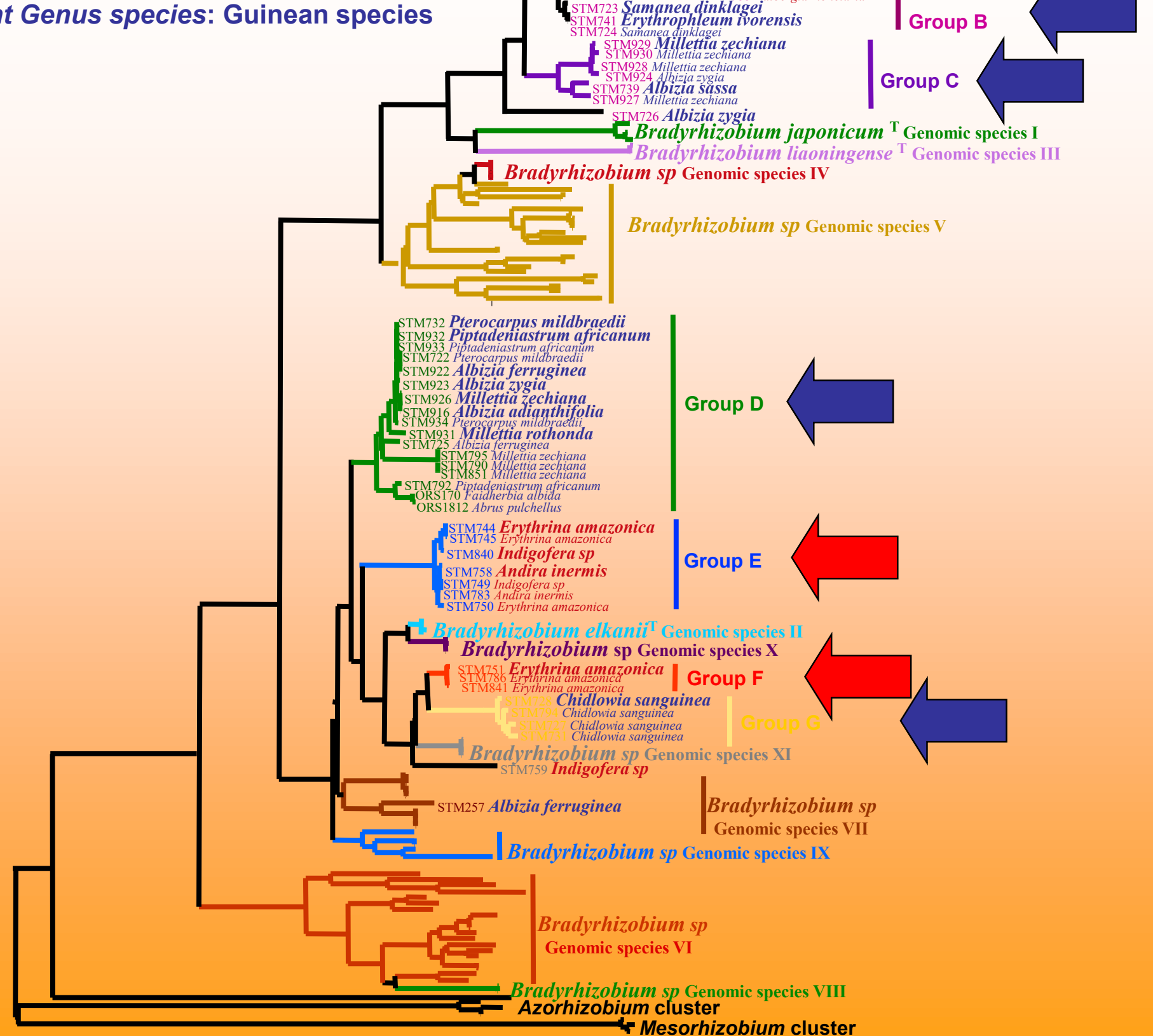




\section{Inoculation trials in Republic of Guinea}

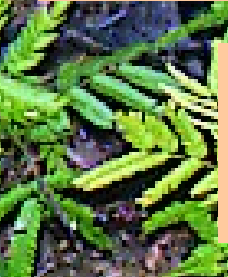

\section{- Inoculation in nursery (50 replicates)}

-Transplantation to the field after 3 months

- Field growth response after 4 months

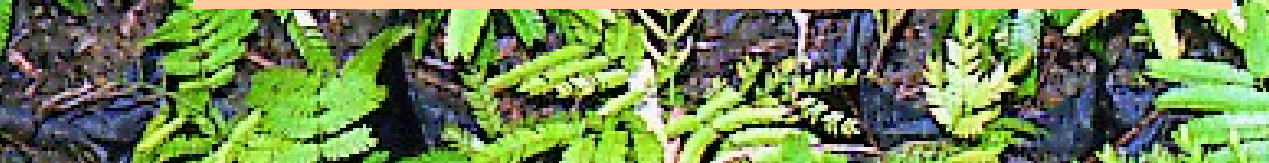

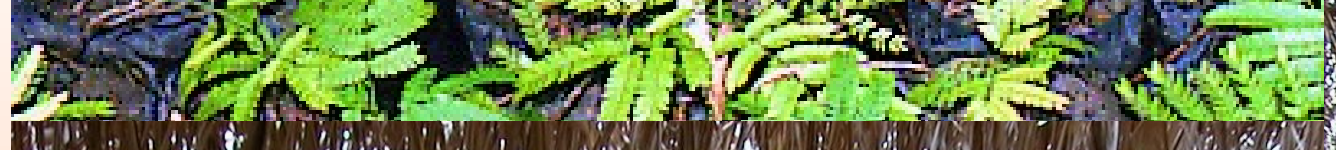

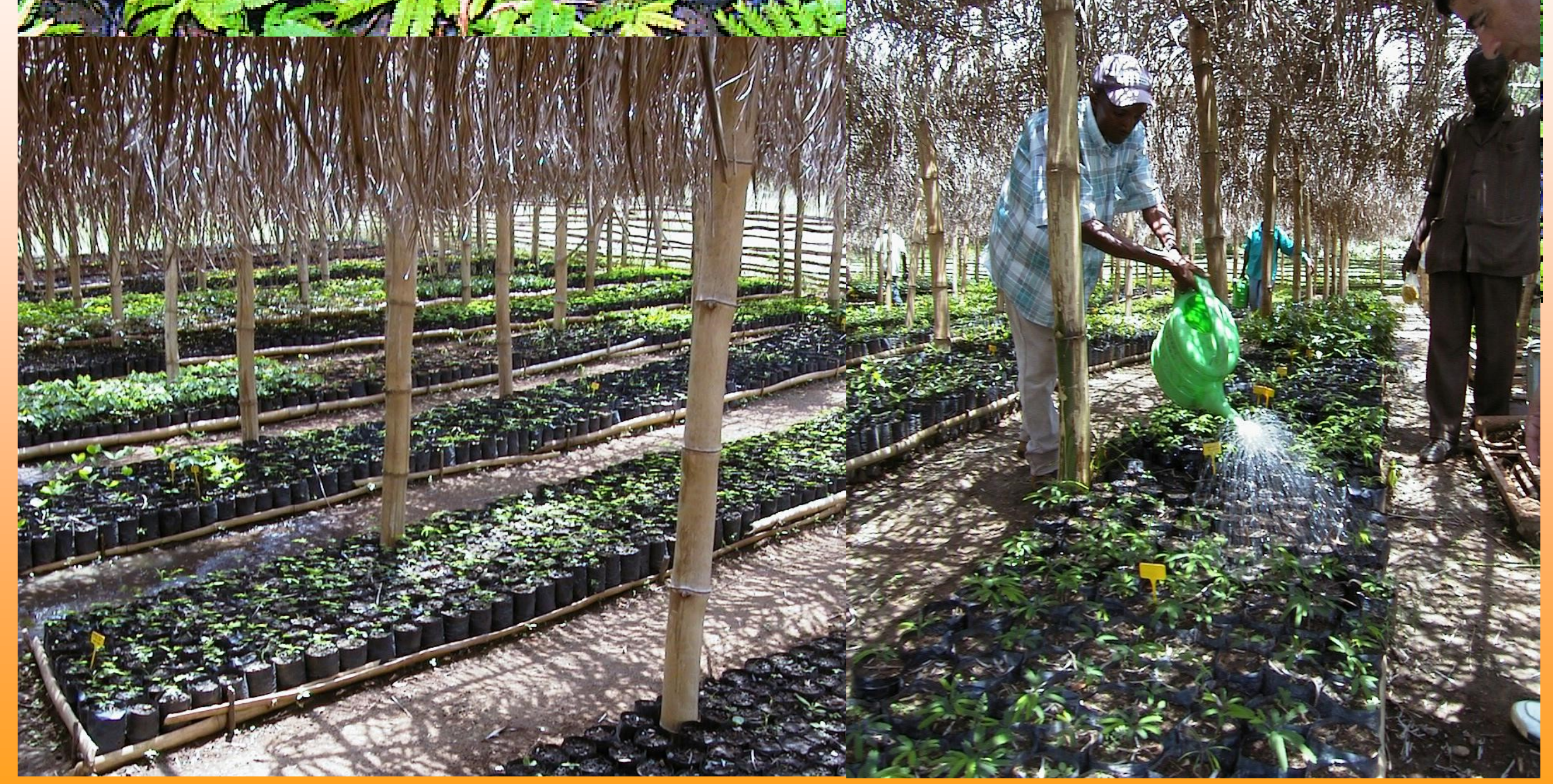


Effect of rhizobial inoculation on growth of Albizia spp. and Samanea dinklagei (after 4 months)

Height

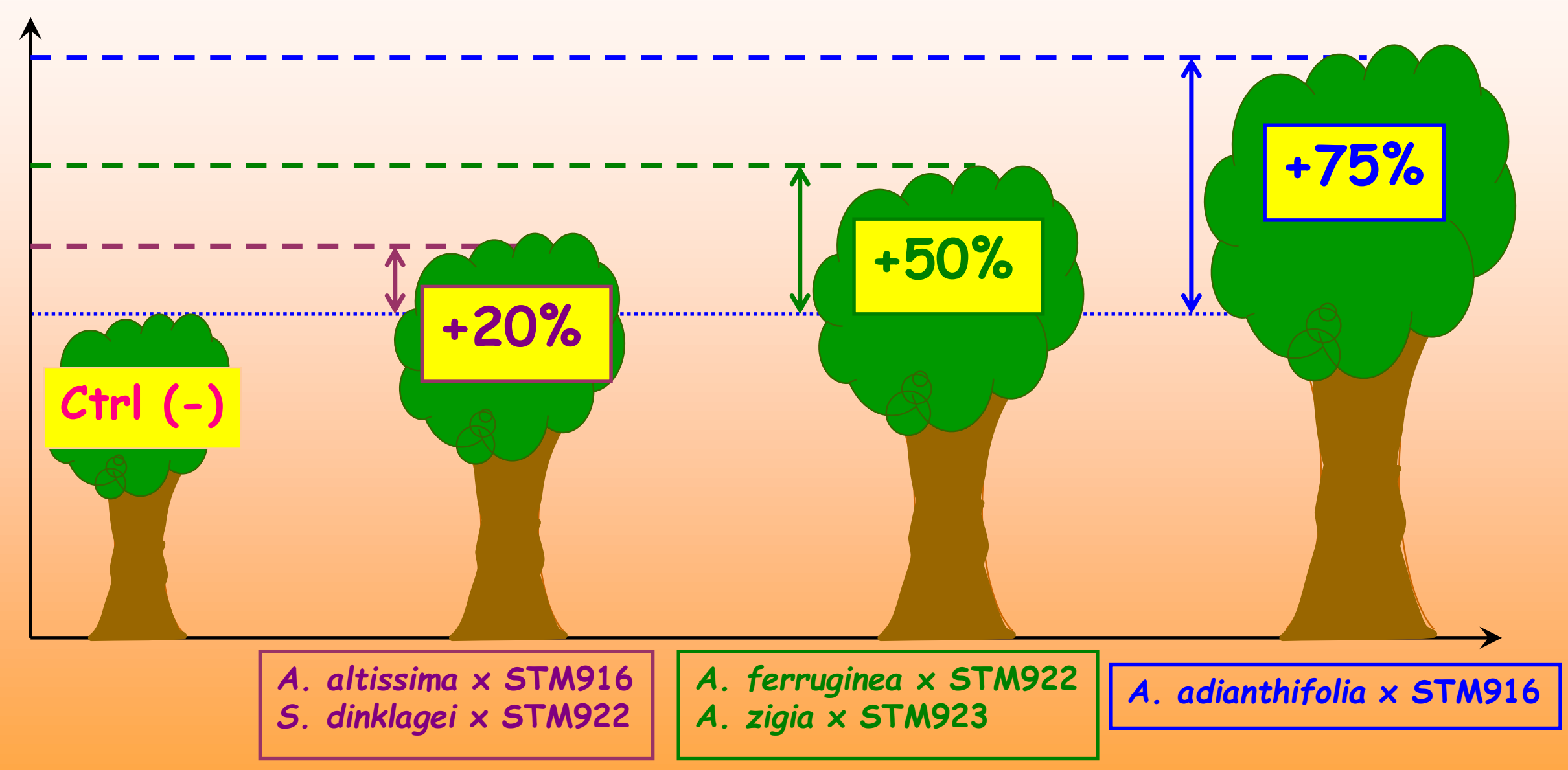


Effect of rhizobial inoculation on growth of Millettia spp. (after 4 months)

Height

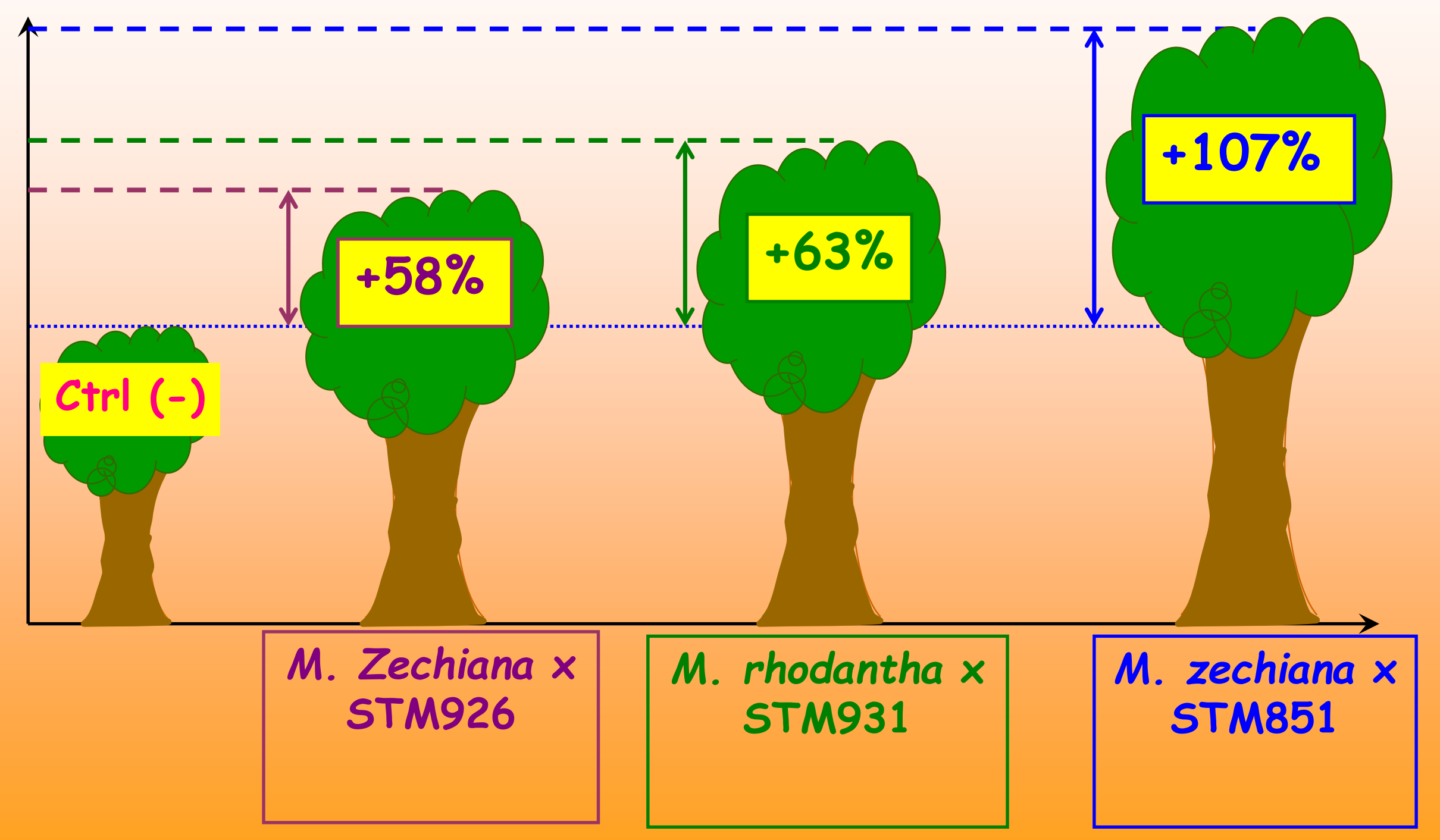


Effect of rhizobial inoculation on growth of Erythrophleum spp. (after 4 months)

Height

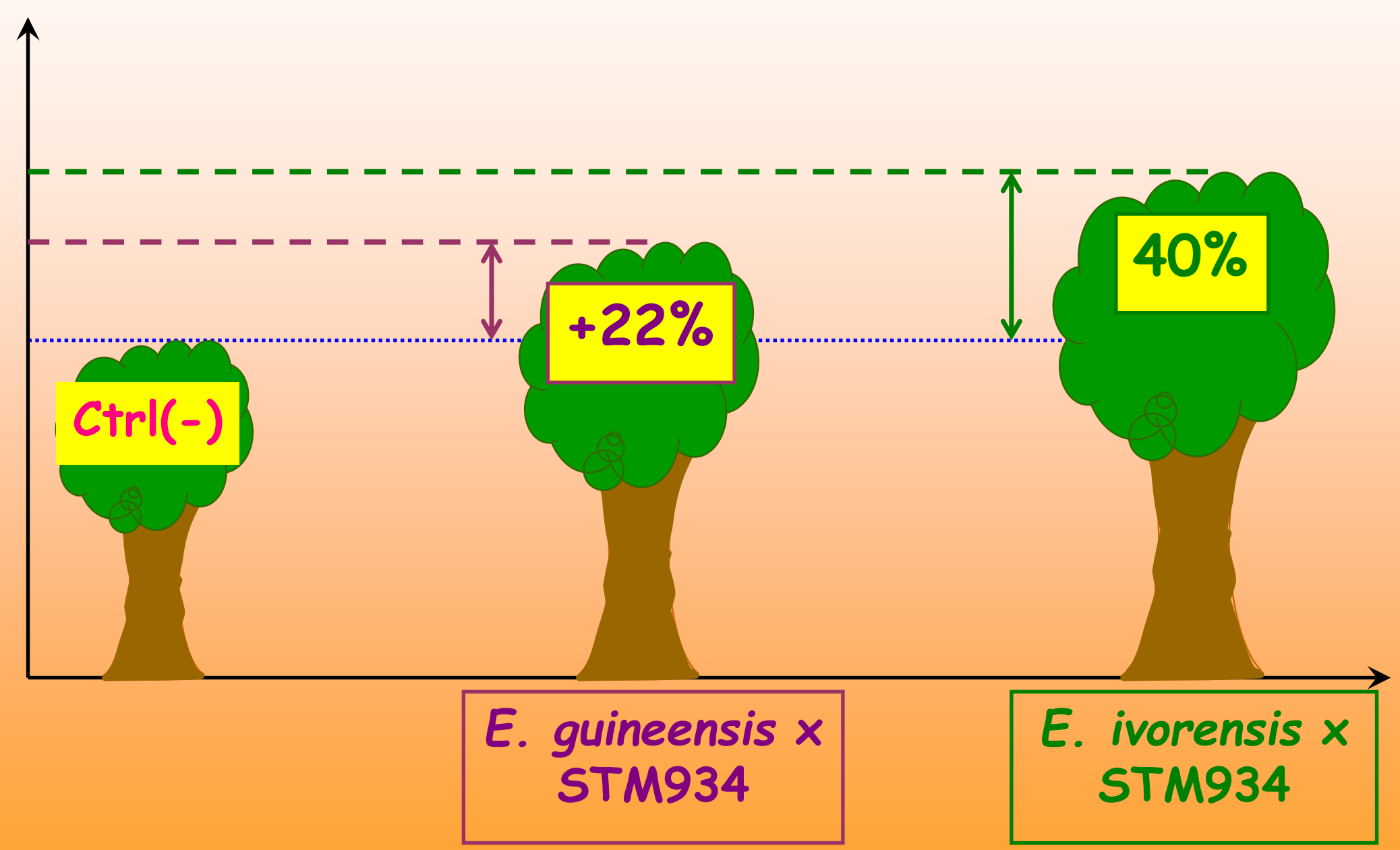




\section{CONCLUSION}

- Tropical rainforests $=$ huge reservoirs of symbioses

- Predominance of the genus Bradyrhizobium

- Need for collaborative research between plant and bacteria phylogenists

- Large potential of applications for reforestation with endangered species 


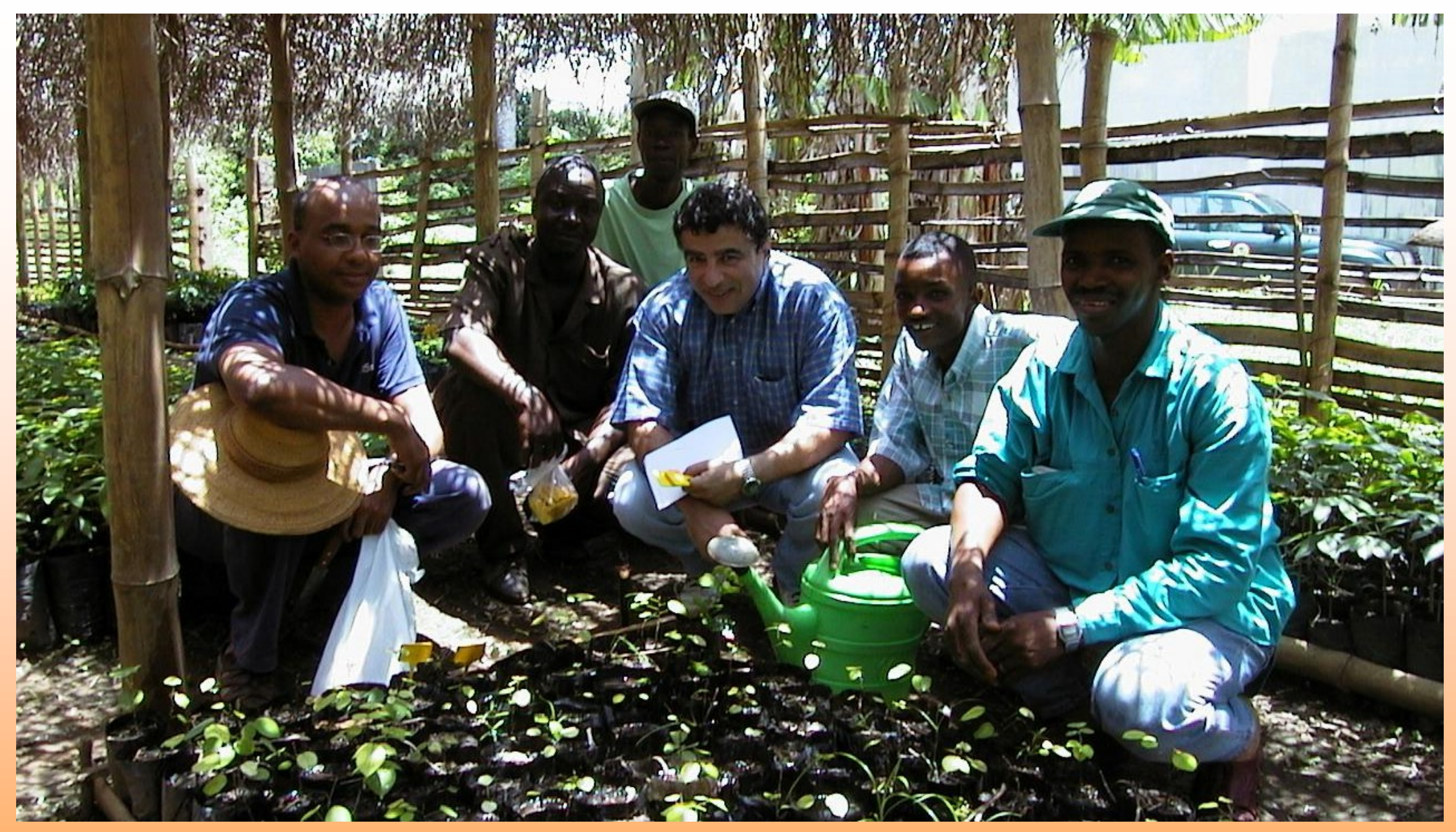

- IRAG, BP 1523, Conakry, République de Guinée

- Universita Autonoma del Puebla, Mexico

- Embrapa/Agrobiologia, BR465, Seropedica, Brazil

- LSTM, Montpellier, France 